\title{
A INSERÇÃO DA HOTELARIA NOS HOSPITAIS PÚBLICOS
}

\author{
Matheus Mendes Pascoal ${ }^{1}$ \\ Vanieli de Souza ${ }^{2}$
}

RESUMO: Hoje há uma grande busca pela qualidade de saúde, humanização do atendimento, conforto para os pacientes, familiares e equipe de profissionais, dessa forma, a implantação do serviço de hotelaria nos hospitais tem sido um recurso que adotaram para aliar ao tratamento e assistência, conciliando o objetivo do hospital com a hotelaria. Diante disso, este trabalho tem por objetivo realizar uma pesquisa bibliográfica até o ano de 2018, para verificar sobre a inserção da hotelaria nos hospitais públicos, analisando os possíveis benefícios da hospitalidade para os pacientes, familiares e profissionais da saúde. Foram analisados artigos brasileiros originais e de revisão onde foi possível encontrar artigos publicados do ano de 2003 até 2016 para a elaboração do presente trabalho, onde abordavam sobre a preocupação dos hospitais em buscar novos recursos no empreendimento hoteleiro, para se adaptar as novas exigências do mercado obtendo maior qualidade no serviço, mas para isso cada vez mais os hospitais vêm se obrigando a desenvolver um novo perfil de atendimento para seus pacientes e equipe. Conclui-se então que a inserção da hotelaria gera muitos benefícios, contudo há uma necessidade de melhorias para otimização do mesmo, necessidade que ocorre não por falta de interesse dos gestores, mas sim de conhecimento do assunto e o seu potencial que o setor oferece aos pacientes, e por se tratar de uma área relativamente nova faz-se necessário aprofundamento desde estudos para futuras pesquisas.

Palavras-chave: Hotelaria hospitalar. Hospitalidade. Qualidade.

\footnotetext{
${ }^{\mathrm{I}}$ Graduando em Enfermagem-Centro Universitário Integrado. Editor Júnior da Sabios, Membro da LAESM e LADCIST.E-mail:matheus_mendesıs@hotmail.com.

${ }^{2}$ Graduanda em Enfermagem-Centro Universitário Integrado, membro da LADCIST e LAACENF.
} 


\section{INTRODUÇÃO}

Antigamente na história humana, não existia um local específico para acolher as pessoas para que elas realizassem seus tratamentos, foi então que no início da era cristã surgiu do latim a palavra hospitalis, termo que atualmente conhecemos por hospital, local este, que se destinam a hospedar pessoas por um período breve na qual irão se submeter a algum tipo de tratamento. Quando falamos em hospedagem, é necessário entendermos sobre hospitalidade, e que apesar do conceito de hospitalidade ser antigo, a hotelaria nos hospitais só surgiu para fazer parte desse conjunto a pouco mais de dez anos, sendo considerada recente nesse meio (RIBEIRO, 2013).

Hoje há uma grande busca pela qualidade de saúde, humanização do atendimento, conforto para os pacientes, familiares e equipe de profissionais. Dessa forma, cresceu-se então a preocupação de adotar melhorias nas instalações, atendimento, segurança, qualidade de serviço e isso tem se tornado um diferencial nos hospitais a fim de tornar a estada do paciente mais agradável (SOUZA, 2006; RIBEIRO, 2013). Por não se tratar apenas de uma opção institucional, mas de uma exigência do mercado concorrente e de seu público específico, é uma grande tendência de mercado (RIBEIRO, 2013).

Segundo Almeida (2009), a implantação do serviço de hotelaria nos hospitais tem sido um recurso que adotaram para aliar ao tratamento e assistência, conciliando o objetivo do hospital com a hotelaria. Ribeiro (2013) e Marques e Pinheiro (2009) ressaltam que o investimento no conforto de suas instalações, na infraestrutura, nos serviços e nos treinamentos é humanizar os serviços, voltado para a cura, para a saúde, sugerindo que tanto o paciente quanto o seu acompanhante, sintam-se como se estivessem hospedados em um hotel e não nas dependências de um hospital.

Pode se dizer então que não é apenas uma estrutura física, mas sim uma proposta de trabalho que inclui o conceito de hospitalidade em todas as ações empregadas no dia a dia de um hospital. Embora o foco dos hospitais sejam tratamento e assistência, implantar esse conceito faz com que comecem a investir em serviços que envolvam valores, modelos e ações com a finalidade de amenizar pensamentos negativos dos pacientes, proporcionando a eles e seus familiares um total conforto, segurança e bem-estar, responsabilidade com 
acomodação, roupas, ambiente, higiene, manutenção e alimentação, desde a arquitetura, instalações, equipamentos até os recursos humanos (ALMEIDA, 2009; RIBEIRO, 2013; QUEVEDO, 2004), já Garcia et al., (2016) relata que atualmente estudos confirmam que o bem-estar contribui para a cura do paciente.

Cada vez mais vem sendo inserida no corpo organizacional dos hospitais e no agir dos recursos humanos. Muitos administradores, médicos e empregados de todos os níveis, tocados pela magia da hotelaria, estão procurando interagir com essa proposta que, apesar de recente, está ganhando força no meio hospitalar (RIBEIRO, 2013).

Justifica-se a realização do presente trabalho sobre a inserção da hotelaria nos hospitais, que se pode observar que é um tema que pouco se fala, demandando então mais pesquisa sobre o assunto, sendo assim esse trabalho pretende fornecer dados para eventuais pesquisas sobre a inserção da hotelaria. Este tema foi escolhido porque o termo hotelaria hospitalar tem sido aplicada nos hospitais, uma vez que os mesmos buscam se adaptar à realidade do mercado fornecendo aumentar a qualidade do serviço prestado precisando de mais aprofundamento no assunto. Porém é preciso ainda compreender quais os problemas que os hospitais enfrentam para inserir o serviço de hotelaria.

Diante disso, este trabalho tem por objetivo realizar uma pesquisa bibliográfica até o ano de 2018, para verificar sobre a inserção da hotelaria nos hospitais públicos, analisando os possíveis benefícios da hospitalidade para os pacientes, familiares e profissionais da saúde.

\section{METOdOLOGiA}

Trata-se de uma revisão semi-sistemática da literatura. A busca será realizada na base de dados, Lilacs, e no site Google Acadêmico e Scielo, utilizando as seguintes palavraschave: Hotelaria hospitalar, Hospitalidade, Qualidade. Primeiramente será feito um levantamento bibliográfico onde serão pesquisados para a elaboração deste, artigos brasileiros originais e de revisão, com publicações até 2018. Para a seleção inicial dos artigos, uma leitura previa do título e resumo do mesmo será realizada para melhor refletir qual artigo será utilizado, possibilitando assim, descartar os artigos repetidos e artigos que 
não abordam o tema em questão, tendo como critério de aprovação, artigos que tenham metodologia relacionada ao tema do presente trabalho com a comparação dos autores e suas ideias. Para a obtenção de resultados, serão levantados dados em estudos publicados e estes comparados com outros estudos para que seja então elaborado o trabalho de forma qualitativa.

\section{RESULTADO E DISCUSSÃO}

Foram encontrados artigos publicados do ano de 2003 até 2016 para a elaboração do presente trabalho, sendo localizados 9 artigos publicados no site Scielo, 15 artigos foram localizados no site Google Acadêmico, e 7 na base de dados Lilacs que se referiam sobre hotelaria hospitalar. Destes, foram utilizados 17 artigos, sendo que o critério de seleção foi abordar sobre a inserção da hotelaria nos hospitais. Foram excluídos os temas voltados a gastronomia hospitalar entre outros assuntos que não abordava o tema em si.

O processo de formação da sociedade foi impulsionado pelos próprios deslocamentos realizados pelo homem, nos mais variados tempos (OLIVEIRA et al., 2012), onde essas constantes mudanças e competitividade contemporânea no mercado da saúde levaram a organizações públicas e privadas a se preocuparem com a qualidade dos serviços prestados na tentativa de agradar os clientes de forma ampla (FREIRE et al., 2013).

Com a globalização o processo de comunicação tornou-se mais fácil, e as pessoas hoje estão mais informadas e mais exigentes, difíceis de serem agradados e mais preocupados com a qualidade (SOUZA, 2006; FREIRE et al., 2013).

Então a preocupação por buscar novos recursos para melhoria cresceu, e o negócio de empreendimentos hoteleiros aumentou significativamente. Porém para se adaptar as novas exigências do mercado, obtiver maior qualidade, algumas iniciativas devem ser tomadas, entre elas são: Melhoria nos equipamentos e instalações, padronização dos serviços, investimentos em recursos humanos, levando uma agilidade no serviço, auto índice de satisfação e hospitalidade (SOUZA, 2006).

Cada vez mais essas características vêm despertando o interesse dos hospitais, que pelas exigências atuais do mercado, vem se obrigando a desenvolver um novo perfil de 
atendimento para seus pacientes e equipe. Os pacientes desse ramo priorizam vantagens como segurança, conforto e bem-estar, ou seja, eles estão em busca de bem mais que um tratamento médico eficiente, já que cada dia mais eles sabem dos seus direitos e exigem obtê-los (FREIRE et al., 2013; GONÇALVES e FERREIRA, 2013; RIBEIRO, 2013; SOUZA, 2006).

Nesse contexto, no estudo feito por Garcia et al., (2016) ele relata que os clientes da área da saúde avaliam os benefícios que as instituições de saúde têm a lhe oferecer e preocupam-se ainda mais com o tipo de atendimento que receberão, optando por ambientes que demonstrem hospitalidade, solidariedade, respeito pelo seu estado físico e emocional.

E por sua vez os hospitais buscam no exemplo de qualidade oferecido pela hotelaria extrair conceitos e serviços que contribuam para aperfeiçoar o atendimento hospitalar, a fim de tornar a estada do cliente de saúde e o ambiente hospitalar mais agradável uma vez que o cuidado na qualidade dos serviços prestados é uma necessidade básica (GONÇALVES e FERREIRA, 2013; RIBEIRO, 2013; SOUZA, 2006).

Souza (2006) ressalta que diante desses fatos os hospitais começaram a implantar os serviços de hotelaria que constam da inserção de conceitos e serviços existentes num hotel devidamente adaptados a um hospital, com a denominação de hotelaria hospitalar, porém, para Batista (2005) a hotelaria hospitalar sempre existiu no ambiente hospitalar só que de forma sutil, já segundo Ribeiro (2013) destaca que é uma nova tendência a hotelaria hospitalar onde agrega ciência, tecnologia, conforto e segurança na hospitalidade, oferecendo para os pacientes qualidade, valor e satisfação, contribuindo no aprimoramento hospitalar, que deve ser o resultado de matéria, trabalho e valores.

Segundo Ribeiro (2013), o conceito de hotelaria hospitalar pode se dizer que surgiu das diferentes necessidades do paciente quando passaram a fazer questionamentos sobre suas necessidades com relação a oferta da cura, entre muitos outros fatores, no entanto os questionamentos foram os motivos mais relevantes para o surgimento desse serviço nos hospitais. A hotelaria hospitalar não é sinônimo de luxo, mas sim de conforto, onde atende todas as necessidades do paciente, respeitando os valores éticos e culturais, com o máximo de confidencialidade de toda e qualquer informação pessoal. 
Para Boeger (2003), a hotelaria hospitalar compreende "a reunião de todos os serviços de apoio, que, associados aos serviços específicos, oferecem aos clientes internos e externos conforto, segurança e bem-estar durante seu período de internação”.

Para implantar o serviço de hotelaria no ambiente hospitalar, os hospitais tanto públicos quanto privados, se faz necessário estudar e ter habilidades para tal serviço, já que a hotelaria hospitalar se preocupa em atender bem o cliente desde a recepção com internações e altas, como também o serviço de quarto, a higiene do ambiente, nutrição, rouparia, lavanderia, agendamento, e por ser um conceito novo algumas instituições ainda não estão preparadas (RIBEIRO, 2013; SILVA e JARCZEWSKI, 20II).

Outro grande fator que pode contribuir de forma positiva na implantação da hotelaria hospitalar, é o desempenho dos funcionários em relação ao seu comportamento, isso por que o mau humor entre outros comportamentos pode ser transmitido para os pacientes (SILVA, 2013).

A hospitalidade oferecida pelos hospitais é um serviço no qual consegue muitas vezes tornar um momento de fragilidade de um paciente em segurança, trazendo a tranquilidade e bem-estar que ele necessita naquele exato momento, através da qualidade de estrutura física e outros serviços disponíveis, já que por sua vez um hospital é procurado exclusivamente quando essas pessoas necessitam de algum tipo de atendimento médico para a realização de seus tratamentos, isso porque muitas delas têm a visão de um estabelecimento que origina tristezas, medos e angustias (GONÇALVES e FERREIRA, 2013; RIBEIRO, 2013; SOUZA, 2006).

Cada paciente tem suas necessidades, um idoso de acordo com Almeida (2009), fazse necessário conhecer como são seus hábitos, seus costumes e até mesmo qual a sua religião, para que ele se sinta em um ambiente seguro, já que por sua vez, esses pacientes possuem maior fragilidade podendo ser ela física e emocional. $O$ serviço de hotelaria hospitalar nesses casos deve prestar muita atenção quanto a horário, tipo de alimento a ser distribuída, rotina de banho entre outros procedimentos.

Por exemplo, em seu estudo, Almeida (2009) relata que os pacientes idosos de Macapá-AP possuem costumes de dormir em redes, ter um recipiente no qual utilizam 
cuspir entre outros hábitos que foram deixados nos quais eles são acostumados, assim para que a estadia em um hospital seja menos traumática, é importante que os funcionários respeitem isso. É possível acreditar que fornecer algo pessoal para o paciente durante sua estadia no hospital auxilia na cura.

É uma tendência nos hospitais na qual tenta retirar aquele estereótipo de lugar frio, triste, onde não há preocupação com o próximo, oferecendo calor humano a estadia dos pacientes internados e os acompanhantes, para que esse momento seja menos traumático possível, priorizando não apenas a infraestrutura do ambiente, mas sim a humanização dos serviços (GONÇALVES e FERREIRA, 2013; RIBEIRO, 2013; SOUZA, 2006).

No Brasil a hotelaria hospitalar é recente com um pouco mais de uma década e tem evoluído nas regiões (GONÇALVES E FERREIRA, 2013; RIBEIRO, 2013; OLIVEIRA et al., 2012), porém de acordo com Gonçalves e Ferreira (2013) e Oliveira et al., (2012) sua frequência está mais presente em hospitais particulares por uma questão financeira, por ter condições de contar com equipamentos de alta tecnologia e profissionais qualificados, já nos hospitais públicos no Brasil, devido à ausência de gestão e investimentos adequados, torna esse serviço menos frequentes a esse segmento.

É possível observar que no Brasil ultimamente a saúde pública passa por sérios problemas decorrentes de falta de investimentos. Uma pesquisa do Instituto Brasileiro de Geografia e Estatística (IBGE, 2010) verificou que o número de leitos para internação caiu de 2,4 para 2,3 por mil habitantes, de 2005 a 2009, deixando abaixo do indicado pelo ministério, entre 2,5 e 3. O resultado foi que o Brasil perdeu II.214 leitos, uma queda de 2,5\% (SILVA, 2013).

Ainda há aqueles hospitais que seu quadro de profissionais não está completo, faltam, médicos, enfermeiros, assistentes sociais, e também a falta de leitos, e isso faz com que haja deficiência no atendimento, causando transtornos para os pacientes e sobrecarga de serviços para os funcionários fazendo com que muitos trabalhem de forma insatisfatória. Nesses casos, a gestão do hospital tem que trabalhar para que haja sempre melhorias no sistema de saúde de diferentes cidades do Brasil (SILVA, 2013). 
"A ausência de qualificação profissional é um dos grandes problemas desse setor, pois são poucas as atividades, que não precisam de criatividade e capacitação" (MAMEDE e NETO, 20II).

Ressalta Silva (2013), que em hospitais da rede pública, o serviço de hotelaria hospitalar é visto como uma área de grandes transformações, sendo de grande relevância para a instituição, que receberam muitos benefícios com o serviço prestado, contudo, o paciente é o que mais ganha benefícios desse serviço, onde se sentirá mais confortáveis na sua estadia, mesmo o hospital não tendo uma estrutura adequada.

Em muitos hospitais a demora no atendimento devido às filas, falta de recursos como o de estrutura, aparelhos especializados, até mesmo em profissionais, faz com que muitos usuários da rede pública, sintam-se desmotivados em procurar por atendimento, e por isso vire alvo de críticas. Por outro lado, acredita-se que um simples gesto por parte da equipe, pode mudar muitos conceitos e fazer a diferença no tratamento do paciente durante sua permanência (MASCARENHAS e SOUZA, 2015).

Contudo, diante das necessidades dos hospitais públicos no Brasil, Oliveira et al., (2012) descreve que: “apesar de muitos hospitais públicos enfrentarem dificuldades adicionais se comparados com hospitais privados, há algum deles que conseguem, com criatividade e competência, desenvolver trabalhos tão bons quanto os de alguns hospitais privados”, ou seja, com uma gestão eficiente, desenvolvendo ótimos trabalhos, conseguirão atingir os objetivos da humanização, primeiro passo para se ter uma hotelaria hospitalar.

Porém faz-se necessário ainda analisar melhor sobre a questão estrutural da saúde pública e privada no Brasil, pois ainda não é possível ter nos hospitais públicos uma apropriada humanização de serviço (OLIVEIRA, et al., 2012).

Em um estudo realizado por Silva e Jarczewski (20II), mostra que a gestora da Casa de Saúde da Cidade de Santa Maria-RS, busca realizar diversas melhorias no hospital em relação a hotelaria hospitalar, porém a situação dos hospitais públicos é diferente, porque eles necessitam que o poder público invistam mais nesse serviço, contudo, a gestora relata que implantando a humanização e hospitalidade, melhorando o acolhimento, tratando 
todos da mesma maneira tanto os pacientes quanto familiares, já é possível fazer um diferencial no hospital mesmo sem recursos.

Ainda em seu estudo foi perguntado para a gestora se ela acreditava que a implantação da hotelaria hospitalar contribuía de forma positiva para o hospital, e sua resposta foi que: "Acho que sim. Acho que tudo que é bom pro hospital é bom pro resto. Contribui pro contentamento. A pessoa já vem pra dentro do hospital psicologicamente abalada, tanto o doente, como a família e encontra tudo ruim”. (SILVA E JARCZEWSKI, 20II).

O Ministério da Saúde tem pressionado as instituições, por meio de órgãos como a Agência Nacional de Vigilância Sanitária (ANVISA), divulgando anualmente uma série de regulamentações voltadas para a segurança do cliente hospitalar, e sobre, a inadequação nos serviços hospitalares, a mídia tem ajudado a população nos últimos anos, com a divulgação de reportagens e denúncias na qual deixam mais atentas a população sobre possíveis irregularidades nesse setor (FREIRE et al., 2013).

De acordo com Vieira (2004), a qualidade nos serviços apresenta dez princípios, que são: Total satisfação do cliente; Gerência participativa; Desenvolvimento de recursos humanos; Constância de propósitos; Aperfeiçoamento contínuo; Gerência de processos; Delegação; Disseminação de informações; Garantia da qualidade; Não aceitação de erros (erro zero).

Oliveira et al., (2012), observa a necessidade na adequação de investimentos nos quais melhorem a qualidade do atendimento e entre outros serviços prestados e não somente insiram dinheiro sem saber seu destino final, ressalta também a necessidade de se ter uma equipe que esteja capacitada para a realização do mesmo, porém segundo Freire et al., (2013), demonstra que tanto as instituições hospitalares públicas como as privadas têm investido em melhorias da qualidade da assistência prestada, da estrutura física e dos processos de trabalho, a fim de atingir as expectativas atuais e futuras dos clientes.

Segundo Silva e Jarczewski (20II), muitos hospitais contam com chefes de cozinha, lojas, salão de beleza, recepção diferenciada, controle de infecção e higienização adequadas, áreas de lazer, salas de espera e acomodações para acompanhantes com maior conforto, 
bancos 24 horas, floricultura, lojas de conveniências, academia, música ao vivo, salas de recreação para crianças e muito mais.

Para que se possa implantar o serviço de hotelaria hospitalar, é necessário que se conheça a instituição, isso porque cada instituição trabalha de forma diferente, sendo assim a implantação desse serviço irá ocorrer de diferentes formas de acordo com a necessidade de cada hospital no qual será aplicado, no entanto, o hospital que utiliza desse conceito precisa ter novos planejamentos para se adaptar sem que perca sua essência desde a estrutura física até a equipe gestora (SOUZA, 2006).

De acordo com Oliveira et al., (2012), para obter uma arquitetura hoteleira hospitalar de qualidade, se faz necessário passar por certas adaptações na estrutura e também profissional, passando por mudanças, isso porque não basta ser belo, tem que ter praticidade e agilidade para que se tenha bons resultados de funcionamento.

O ambiente do paciente hospitalizado, não pode ser mais limitado como antigamente, como por exemplo, proibir de sair do seu quarto. Segundo o Ministério da Saúde o ambiente segue três eixos (ALMEIDA, 2009):

- Um espaço que possibilite a reflexão da produção do sujeito e do processo de trabalho.

- A confortabilidade focada na privacidade e individualidade dos sujeitos envolvidos, exaltando elementos do ambiente que interagem com o homem como cor, cheiro, som, iluminação e garantam a trabalhadores, pacientes e sua rede social.

- O espaço como ferramenta facilitadora do processo de trabalho funcional favorecendo a otimização de recursos e o atendimento humanizado, acolhedor e resolutivo.

Nesse contexto, existem pequenos processos a seguir que podem fazer a diferença no espaço para a harmonia do ambiente e agradar mais os pacientes (ALMEIDA, 2009).

- A iluminação natural garante ao paciente saber como está o tempo la fora, dia/noite, chuva/sol, já a iluminação artificial, além da ajuda na realização das atividades, deixa um ambiente mais aconchegante. 
- A música ambiente é muito favorável nesses casos, trazendo paz, tranquilidade para os pacientes, deixando o ambiente mais calmo, mais alegre.

- As cores utilizadas estimulam os sentidos e podem encorajar ao relaxamento, ao trabalho, ao divertimento ou ao movimento.

- Ambientes externos com jardins e áreas com bancos para que os funcionários e familiares possam se acomodar e descansar, é um grande aliado no bem-estar nos hospitais.

- Individualidade, pois cada paciente é diferente um do outro.

- Uso de divisórias e cortinas para a privacidade dos pacientes.

É possível hoje com a prática da hotelaria hospitalar, gerar lucros, se bem planejados, pois apesar do crescimento da tecnologia muitos hospitais ainda não possuem uma visão voltada para esse serviço, entretanto os pacientes estão ficando cada vez mais exigentes, e isso faz com que o setor hoteleiro nos hospitais precise se aprimorar (OLIVEIRA et al., 2012).

Existe um método para obter melhor resultados nas empresas que pode ajudar na progressão da empresa, chamada de método PDCA, esse método consiste em um planejamento estratégico buscando crescimento da empresa (SILVA, 2013).

- Planejamento: Estabelecimento de metas para cada item de controle/identificação de problemas;

- Execução: Executar as tarefas exatamente como previstas na etapa do planejamento. Para tal é preciso educar e treinar as pessoas no trabalho. Havendo coleta de dados para próxima etapa.

- Verificação: Avaliar e monitorar os resultados obtidos;

- Ação: Agir de acordo com o observado, conforme os relatórios e diagnósticos aperfeiçoando o desempenho e corrigindo falhas casuais.

Por ser o hospital uma instituição complexa de ser administrada, para que haja um ótimo funcionamento do serviço de hotelaria nos hospitais, o trabalho em equipe é fundamental para a melhoria na comunicação da equipe hospitalar, envolvendo todos os departamentos juntamente com a gerência, tendo a necessidade de muitas vezes investir 
no quadro de funcionários, mudanças de comportamento, até mesmo de mentalidade, para que então juntos possam estar buscando a humanização dos serviços e com isso obter para os pacientes um serviço de alta qualidade, afinal a gestão precisa estar aberta e atenta às mudanças, ser pessoas comprometidas e inovadoras, buscando sempre atingir resultados com inovação e flexibilidade, conseguindo desse modo, estar à frente dos acontecimentos nesse tipo de serviço que sempre muda repentinamente, a final é necessário evoluir (BATISTA, 2005; GONÇALVES e FERREIRA, 2013; RIBEIRO, 2013; SOUZA, 2006).

Souza (2006) e Quevedo (2004) relatam que o foco dos hospitais não deve ser restrito apenas com os pacientes, mas também, dar uma boa comodidade aos acompanhantes e visitantes, sem esquecer-se dos médicos e outros profissionais que contribuem para o funcionamento em geral, afinal a hospitalidade depende de todo o quadro de equipe desde a chegada do paciente e acompanhante até a sua saída, e de acordo com Garcia et al., (2016) pode-se afirmar que a relação entre instituição e paciente colabora para a evolução do tratamento.

E diante desse fato, SILVA e JARCZEWSKI (20II), ressalta que independente da classe social da pessoa, ela merece e deve ser bem acolhida e tratada por todos do hospital, sem nenhum tipo de discriminação.

Para ter sucesso nesse ramo de hotelaria hospitalar, é preciso que o serviço encante os pacientes e familiares, que seja um ambiente agradável com cores claras, ou seja, um ambiente para que essas pessoas possam se sentir confortável com pessoas dispostas a trabalhar de forma entusiasmada (BARBOSA, et al., 2013).

Gonçalves e Ferreira (2013), afirmam que apesar de todas as dificuldades de implantar o serviço de hotelaria nos hospitais, essa prática garante ótimos resultados, medidos pela satisfação dos pacientes. E para que haja satisfação dos mesmos, é necessário um serviço de qualidade, segundo Souza (2006) é importante salientar que tudo se trata de uma adaptação, pois é necessário compreender que o ambiente hospitalar apresenta maior complexibilidade em relação ao hoteleiro. Ainda é possível observar nos tempos de hoje que muitos hospitais devido à falta de um bom planejamento carecem da atenção (SILVA, 2013). 


\section{CONCLUSÃO}

A inserção da hotelaria nos hospitais gera muitos benefícios tanto para os pacientes como também para o próprio hospital, contudo há uma necessidade de melhorias para otimização do mesmo, necessidade que ocorre não por falta de interesse dos gestores, mas sim pela falta de conhecimento acerca do assunto ou até mesmo muitas vezes pela falta de recursos, e por se tratar de uma área relativamente nova faz-se necessário aprofundamento de estudos para futuras pesquisas, pois ainda se tem poucas publicações sobre o assunto, e isso contribuirá de forma modesta no desenvolvimento da hotelaria hospitalar no Brasil.

\section{REFERÊNCIAS}

ALMEIDA, M. N. B. C. Hotelaria Hospitalar: Cultura e hospitalidade no atendimento a idosos. 2009. 63 f. Monografia (Especialização em Hotelaria Hospitalar). Universidade de Brasília, Centro de Excelência em Turismo, Brasília, 2009.

BARBOSA, J. G. et al. Hotelaria hospitalar: novo conceito em hospedagem ao cliente. Cogitare Enfermagem. Curitiba, v. 18, n. 3, jul/set, 2013.

BATISTA, M. E. C. Humanização em hospitais por meio da hotelaria: uma forma de agregar valor à administração hospitalar. 2005. 5of. Monografia (Especialização no Curso de Formação de Consultores em Turismo). Universidade de Brasília, Centro de Excelência em Turismo, Brasília, 2005).

BOEGER, M. A. Gestão em Hotelaria Hospitalar. São Paulo: Atlas, 2003.

FREIRE, E. M. R. et al. Hotelaria hospitalar: desenvolvimento desde conteúdo em cursos de graduação em enfermagem. Convibra. Alfenas/MG, p. I-6, 2013.

GARCIA, I. F et al. Humanização na hotelaria: um diferencial no cuidado com o paciente. Revista Saúde e Desenvolvimento. Curitiba, v. ıo, n. 5, p. 196-207, jul/dez, 2016.

GONÇALVES, I. C. O; FERREIRA, L. V. F. Gestão de hotelaria hospitalar: Percepções e fundamentos. Revista Turismo: Estudos e Práticas. Mossoró/RN, v. 2, n. 2, p. 154-173, jul/dez, 2013.

MAMEDE, M. I. B; NETO, A. R. V. Qualidade percebida e expectativas de brasileiros e estrangeiros em relação aos equipamentos e serviços Turísticos. Revista Turismo Visão e Ação - Eletrônica, v. 13, n. 3 - p. 311-328, set/dez., 20II. 
MARQUES, M; PINHEIRO, M. T. A influência da qualidade da hotelaria hospitalar na contribuição da atividade curativa do paciente. Revista Anagrama. v. 2, n. 3, p. I-I5, $\mathrm{mar} / \mathrm{mai}$, 2009.

MASCARENHAS, R. G. T; SOUZA, J. T. A QUALIDADE PERCEBIDA PELO PACIENTE ATRAVÉS DOS SERVIÇOS DA HOTELARIA HOSPITALAR: um estudo sobre a hospitalidade na área da saúde. Turismo e Sociedade. Curitiba, v. 8, n. 3, p. 419-445, set/dez, 2015 .

OLIVEIRA, $\mathrm{C}$ et al. A hotelaria hospitalar como uma nova perspectiva de atuação em organizações de saúde. Revista Turismo: Estudos e Práticas. Mossoró/RN, v. I, n. 2, p. 191209, jul/dez, 2012.

QUEVEDO, M. F. Hotelaria Hospitalar: uma tendência presente nas organizações de saúde. 2004. In: II Seminário de Pesquisa em Turismo do Mercosul. Anais: Universidade de Caxias do Sul, Set/2004, p. I-II.

RIBEIRO, A. B. A hotelaria hospitalar como um diferencial no setor de saúde. Revista Especialize On-line IPOG. Goiânia, v. oI, n. oo6, p. I-I4, dez, 2013.

SILVA, I. C. O. G. PERCEPÇÕES APLICADAS A HOTELARIA HOSPITALAR: análise com gestores em hospitais públicos e privados da Cidade do Natal/RN. 2013. I36f. Monografia (Graduação em Turismo). Universidade Federal do Rio Grande do Norte, Natal - RN, 2013.

SILVA, T. S. P; JARCZEWSKI, M. D. As possibilidades de implantação da hotelaria hospitalar em um hospital público na cidade de Santa Maria - RS. Disciplinarum Scientia.

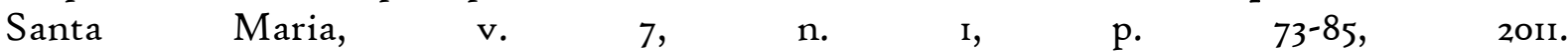

SOUZA, G. G. Hotelaria Hospitalar: Conceitos da hotelaria adaptados ao setor hospitalar. 2006. $84 \mathrm{f}$. Monografia (Graduação em Turismo). Universidade de Minas Gerais, Belo Horizonte, 2006.

VIEIRA, E. V. Qualidade em Serviços Hoteleiros: a Satisfação do cliente é função de todos. Caxias do Sul: EDUCS, 2004 